\title{
A Neural Network Controller for Variable-Speed Variable-Pitch Wind Energy Conversion Systems Using Generalized Minimum Entropy Criterion
}

\author{
Mifeng Ren, ${ }^{1,2}$ Jianhua Zhang, ${ }^{1}$ Ye Tian, ${ }^{3}$ and Guolian Hou ${ }^{2}$ \\ ${ }^{1}$ State Key Laboratory of Alternate Electrical Power System with Renewable Energy Sources, \\ North China Electric Power University, Beijing 102206, China \\ ${ }^{2}$ School of Control and Computer Engineering, North China Electric Power University, Beijing 102206, China \\ ${ }^{3}$ DaTang Technology Industry Group Co., Ltd., Beijing 100097, China
}

Correspondence should be addressed to Jianhua Zhang; zjh@ncepu.edu.cn

Received 10 April 2014; Revised 20 July 2014; Accepted 28 July 2014; Published 12 August 2014

Academic Editor: M. I. Herreros

Copyright (C) 2014 Mifeng Ren et al. This is an open access article distributed under the Creative Commons Attribution License, which permits unrestricted use, distribution, and reproduction in any medium, provided the original work is properly cited.

\begin{abstract}
This paper considers the neural network controller design problem for variable pitch wind energy conversion systems (WECS) with non-Gaussian wind speed disturbances in the stochastic distribution control framework. The approach here is used to directly model the unknown control law based on a fixed neural network (the number of layers and nodes in a neural network is fixed) without the need to construct a separate model for the WECS. In order to characterize the randomness of the WECS, a generalized minimum entropy criterion is established to train connection weights of the neural network. For the train purpose, both kernel density estimation method and sliding window technique are adopted to estimate the PDF of tracking error and entropies. Due to the unknown process dynamics, the gradient of the objective function in a gradient-descent-type algorithm is estimated using an incremental perturbation method. The proposed approach is illustrated on a simulated WECS with non-Gaussian wind speed.
\end{abstract}

\section{Introduction}

With the rapid growth of the global wind industry, wind energy has become one of the most important renewable energy sources [1]. Wind energy conversion system (WECS) technology has undergone rapid development in response to the demands for increasing use of renewable energy [2]. WECSs present two operating modes according to how the wind turbine is connected to the grid. In the fixed-speed mode, the turbine is directly connected to the grid, fixing the rotational speed to the grid frequency. In the variablespeed mode, an electronic converter is inserted between the generator and the grid, or a doubly fed generator (DFIG) controlled by the rotor circuit is used. Thus, the rotational speed can change independently of the grid frequency. In this paper a variable-speed variable-pitch wind energy conversion system is considered. This combination aims to compensate the limitation of each strategy working independently and may improve the transient response and the overall performance.
Control of WECSs is essentially important in terms of energy generation efficiency, power quality, and installation's life. Nevertheless, due to the nonlinearity, uncertainty, and various disturbances that exist in WECSs, it is a challenging problem for controller design. Various control syntheses such as PI regulator [3, 4], optimal control in LQ [5], and LQG form [6] have been developed. These control strategies which use the pitch angle as a control input give acceptable results for rotor speed regulation but showed poor performances in power regulation. In [7], it was shown that the generator torque alone is able to regulate the electrical power in an acceptable way. However, it generates large variations of the rotor speed that are not desirable for the wind turbine structure. Most of the work reported ignores the multivariable nature of WECSs.

Recently, the control of variable-speed variable-pitch WECSs' operation has attracted a lot of attention. A PI controller in the power loop and a self-tuning regulator in the speed loop are proposed in [8]. Considering the 


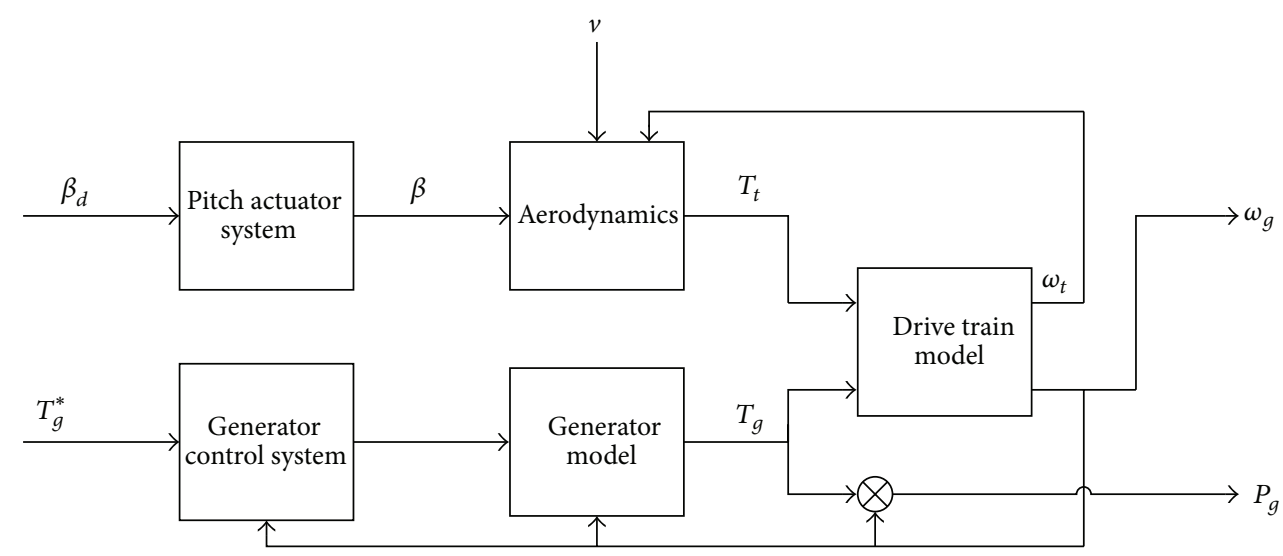

FIGURE 1: A typical variable-speed variable-pitch WECS.

nonlinear and time-varying characteristics of the WECS, advanced control theory and intelligent control scheme have been developed. In [9], sliding mode control is used to cope with system uncertainty and reduce mechanical efforts and chattering. Model predictive control (MPC) is discussed in $[10,11]$, where the constraints on pitch angle and performance specifications can be handled. A gain scheduled $\mathrm{H}$-infinity controller is proposed in [12]. Fuzzy logic based control is an effective approach to address the problem of parameter uncertainties [13]. Based on adaptive subspace predictive control (SPC) method $[14,15]$, investigated the wind turbines control problem in the data-driven framework. However, all these control methods have not fully considered the possible random noises involved in the WECS.

The LQG strategy has been shown to be effective in accommodating plant uncertainties and random disturbance in a systematic and straightforward way in energy conversion control for wind generating systems [16-18]. LQG synthesis design method is based on linear model, and the noises and disturbance are assumed to be Gaussian. However, there are two main problems for WECSs: (1) since it is subjected to nonlinearity and random noises, the accurate model is very complex and even cannot be built; (2) the practical random signal from measurement device and disturbances from wind speed are often of non-Gaussian nature. In this case, the LQG control method may not achieve satisfactory performance. As such, in this study, the entropy of tracking error is employed to characterize the randomness of non-Gaussian WECSs.

Based on the minimum error entropy principle, the stochastic systems with non-Gaussian disturbances can be well controlled $[19,20]$ in the data-driven framework. Since equations governing the system dynamics are unknown, it is very difficult or even impossible to obtain the gradient of the proposed performance function, which is one of the important steps in this method. Therefore, the neural network model is proposed to approximate the unknown nonlinear dynamics firstly, and then the gradient-descenttype control law can be obtained [21]. Without assuming or constructing a separate model for the unknown process dynamics [22, 23], use a neural network controller to directly regulate stochastic systems and the gradient of the objective function is estimated by using the simultaneous perturbation stochastic approximation approach. Motivated by this idea, a neural network controller is proposed for WECSs, where the non-Gaussianity of wind speed disturbances is taken into full account and the control problem is solved in the stochastic distribution control framework. Since the accurate model of WECS is difficult to be established, the neural network controller is designed based on the measurable input and output data according to the generalized minimum entropy principle. An incremental perturbation method is adopted to estimate the corresponding gradient in a gradientdescent-type algorithm. Simulation results show that the proposed minimum error entropy (MEE) control strategy can effectively reduce the influence of the non-Gaussian disturbances from the wind speed.

\section{Problem Formulation}

2.1. System Description and Modeling. A model of the entire WECS can be structured as several interconnected subsystems as shown in Figure 1. The aerodynamic subsystem describes the transformation of kinetic energy stored in the wind into mechanical power via the wind turbine rotor. The drive train subsystem represents the mechanical parts that transfer the aerodynamic torque on the blades to the generator shaft. The pitch actuator subsystem models the pitch control system that controls the pitch angle of the wind turbine's blades. Finally, the electrical subsystem describes the electric generator, the power electronic converters, and the generator control system.

In general, the generator control system is based on a field-oriented vector control strategy where the machine variables are expressed in a synchronously rotating reference frame. Vector control stems from decoupled flux-current and torque-current control in AC drives.

In Figure 1, the input signals coming from the turbine control system are the generator torque set point $T_{g}^{*}$ and the desired pitch angle $\beta_{d}$. The measured outputs are assumed to be the generator speed $\omega_{g}$ and the generator power $P_{g}$. The wind speed $v$ is the disturbance signal affecting the WECS. 


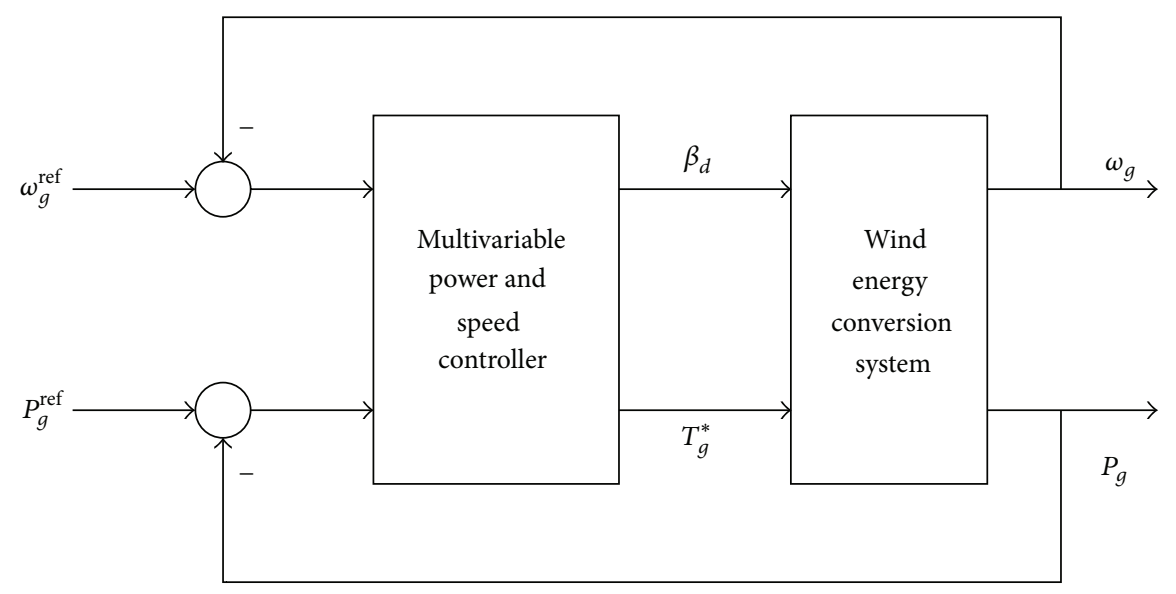

FIGURE 2: The multivariable control scheme.

Denote $x=\left[\begin{array}{lllll}x_{1} & x_{2} & x_{3} & x_{4} & x_{5}\end{array}\right]^{T}=\left[\begin{array}{lllll}\omega_{t} & \omega_{g} & T_{t w} & T_{g} & \beta\end{array}\right]^{T}$, $u=\left[\begin{array}{ll}u_{1} & u_{2}\end{array}\right]^{T}=\left[\begin{array}{ll}T_{g}^{*} & \beta_{d}\end{array}\right]^{T}$, and $y=\left[\begin{array}{ll}y_{1} & y_{2}\end{array}\right]^{T}=\left[\begin{array}{ll}\omega_{g} & P_{g}\end{array}\right]^{T}$. Based on the aforementioned works, the state-space representation of the WECS concerned in this study can be written as [24]

$$
\begin{gathered}
\dot{x}_{1}=-\frac{i}{J_{t}} x_{3}+\frac{1}{J_{t}} \frac{C_{p}(\lambda, \beta)}{\lambda} \frac{1}{2} \rho \pi R^{3} v^{2}, \\
\dot{x}_{2}=\frac{1}{J_{g}} x_{3}-\frac{1}{J_{g}} x_{4}, \\
\dot{x}_{3}=k_{s} \cdot i \cdot x_{1}-k_{s} \cdot x_{2}-\left(\frac{i^{2} \cdot B_{s}}{J_{t}}+\frac{B_{s}}{J_{g}}\right) \cdot x_{3} \\
+\frac{i \cdot B_{s}}{J_{t}} \cdot \frac{C_{p}(\lambda, \beta)}{x_{1}} \cdot \frac{v^{3}}{2} \cdot \rho \pi R^{2}+\frac{B_{s}}{J_{g}} \cdot x_{4}, \\
\dot{x}_{4}=-\frac{1}{\tau_{g}} \cdot x_{4}+\frac{1}{\tau_{g}} \cdot u_{1}, \\
\dot{x}_{5}=-\frac{1}{\tau} x_{5}+\frac{1}{\tau} u_{2}, \\
y_{1}=x_{2}, \\
y_{2}=x_{2} \cdot x_{4} .
\end{gathered}
$$

2.2. Control Problem Description. Designing an effective control system for the WECS is not an easy task. The system variables must be regulated in the presence of severe fluctuations in the input turbine power $P_{t}$ caused by erratic variations in the wind speed. Fluctuations in $P_{t}$ can lead to harmful effects on the system [8]. Large variations in the drive train torsional torque $T_{t w}$ can occur, thus reducing the life time of the mechanical parts of the system. Input power fluctuations can result in electric power fluctuations supplied to the grid. This, in turn, can cause voltage flicker problems and a reduction in the power quality.

Based on the analysis above, the main control objectives in the full load regime are to regulate both the generator power $P_{g}$ and the generator speed $\omega_{g}$ at their rated values $P_{g \text {,rat }}$ and $\omega_{g \text {,rat }}$, respectively. These objectives can be achieved by manipulating the desired pitch angle $\beta_{d}$ and/or the generator torque set point $T_{g}^{*}$. This can be inferred from (2), where the aerodynamic power $P_{t}$ extracted from the wind is determined by the power coefficient, $C_{p}(\lambda, \beta)$. This coefficient can be interpreted as a variable gain controlled by $\lambda$ and $\beta$ :

$$
P_{t}=C_{p}(\lambda, \beta) \frac{1}{2} \rho \pi R^{2} v^{3}
$$

where $\lambda \triangleq \omega_{t} R / v$ is the tip speed ratio and $\omega_{t}$ is the rotor speed of the wind turbine.

Thus, in the full load regime, to regulate the power at its rated value, the power coefficient should be reduced by increasing $\beta$, decreasing $\lambda$, or changing both variables. Consequently, manipulating the pitch angle results in deviations in the power extracted by the wind turbine and, indirectly, induces deviations in the turbine speed via the drive train dynamics. Similarly, the generator torque can affect the turbine speed through the drive train dynamics and can be used for controlling the power extracted by the wind turbine by controlling $\lambda$.

Different from the decentralized control strategy in [25, 26], the multivariable stochastic control approach, shown in Figure 2, is adopted here to establish the WECS control purpose.

The control problem here is solved in the stochastic distribution control framework, and the WECS in (1) is firstly discretized as

$$
\begin{gathered}
x_{k+1}=f\left(x_{k}, u_{k}, v_{k}\right), \\
y_{k}=h\left(x_{k}\right),
\end{gathered}
$$

where $x_{k}=\left[\begin{array}{lll}x_{1 k} & \cdots & x_{5 k}\end{array}\right]^{T} \in R^{5}, y_{k}=\left[\begin{array}{ll}y_{1 k} & y_{2 k}\end{array}\right]^{T} \in$ $R^{2}$ are system state and output vectors, respectively. $u_{k}=$ $\left[\begin{array}{ll}u_{1 k} & u_{2 k}\end{array}\right]^{T} \in R^{2}$ is the control signal applied to the plant. $f(\cdot)$ and $h(\cdot)$ are the system state and output dynamics, respectively. $v_{k} \in R$ is a non-Gaussian bounded random variable with known PDF $\gamma_{v_{k}}$. 
Denote the set point vector of generator speed and generator power by $r=\left[\begin{array}{ll}r_{1} & r_{2}\end{array}\right]^{T}=\left[\begin{array}{ll}\omega_{g}^{\text {ref }} & P_{g}^{\text {ref }}\end{array}\right]^{T}$, and then the tracking error $e_{k}=\left[\begin{array}{ll}e_{1 k} & e_{2 k}\end{array}\right]^{T}$ is

$$
e_{k}=r_{k}-y_{k}=g\left(x_{k}, r_{k}\right) .
$$

The control problem for WECS can be expressed as follows: find proper generator torque set point $T_{g}^{*}$ and the desired pitch angle $\beta_{d}$ such that the generator speed $\omega_{g}$ and the generator power $P_{g}$ can track their set points $\omega_{g}^{\text {ref }}$ and $P_{g}^{\text {ref }}$ as closely as possible in the presence of non-Gaussian wind speed disturbance $v$.

From the above presentation, even though the model of WECS is established under a lot of assumptions and simplifications, the obtained model equations (1)-(4) are still very complex. However, with the development of technology, the input and output data can be easily measured. Therefore, the controller for WECS is designed in the data-driven framework in this paper.

\section{Neural Network Controller Design}

3.1. Formulation of Objective Function. Since the wind speed $v$ is non-Gaussian, the tracking error in (4) is probably nonGaussian. And it is well known that all the randomness information can be characterized by the whole PDF. The main purposes of controller design are twofold: to make the PDF of tracking error follow a narrow and sharp Gaussian-like distribution and to drive the tracking error approaching to zero. Therefore, the chosen objective functions should obey the above two principles.

It is noted that entropy is a general measure of randomness. Minimum entropy of tracking error corresponds to a sharp and narrow PDF; this means that the randomness of the tracking error is minimized. Thus the objective function is

$$
J_{1}=H\left(e_{1 k}\right)+H\left(e_{2 k}\right)+H\left(e_{1 k}, e_{2 k}\right),
$$

where $e_{i k} \in\left[a_{e_{i}}, b_{e_{i}}\right](i=1,2)\left(a_{e_{i}}\right.$ and $b_{e_{i}}$ are lower and upper bounds of the tracking error $\left.e_{i}, i=1,2\right)$, $H\left(e_{i k}\right)=-\log \int_{a_{e_{i}}}^{b_{e_{i}}} \gamma_{e_{i k}}^{2}\left(\tau_{i}\right) d \tau_{i}$ is the quadratic Renyis entropy [27] of each tracking error $e_{i k}(i=1,2)$, and $H\left(e_{1 k}, e_{2 k}\right)=-\log \int_{a_{e_{1}}}^{b_{e_{1}}} \int_{a_{e_{2}}}^{b_{e_{2}}} \gamma_{e_{1 k}, e_{2 k}}^{2}\left(\tau_{1}, \tau_{2}\right) d \tau_{1} d \tau_{2}$ is the joint entropy of tracking errors $e_{1 k}$ and $e_{2 k}$, where $\gamma(\cdot)$ stands for the $\mathrm{PDF}$ of corresponding random variable.

On the other hand, mean value can reflect the magnitude of the tracking error, which calls for another performance function

$$
J_{2}=E\left(e_{1 k}^{2}\right)+E\left(e_{2 k}^{2}\right)
$$

where $E\left(e_{i k}^{2}\right)=\int_{a_{e_{i}}}^{b_{e_{i}}} \tau^{2} \gamma_{e_{i k}}\left(\tau_{i}\right) d \tau_{i}$ is the mean value of each tracking error $e_{i k}(i=1,2)$.

To design the optimal controller for WECS, two objective functions (5) and (6) should be minimized simultaneously.
And the constrained control energy also should be considered. In this paper, the weighting method is used by forming a linear combination of the objectives:

$$
\begin{aligned}
J\left(u_{k}\right)= & \sum_{i=1}^{2}\left(R_{i} H\left(e_{i k}\right)+R_{i+2} E\left(e_{i k}^{2}\right)\right)+R_{5} H\left(e_{1 k}, e_{2 k}\right) \\
& +\frac{1}{2} u_{k}^{T} R_{6} u_{k},
\end{aligned}
$$

where $R_{i}(i=1,2, \ldots, 6)$ are corresponding weights.

Remark 1. Weights in performance index (7) denote the different relative importance of different objectives. The value of the weights usually could be only decided by try-and-error method, based on engineering experiences, repeating simulations, and other information. By parametrically varying the weights in the combined single objective function (7), we could get different optimal control inputs, which are called Pareto optimal solutions. In this paper, repeating simulation approach is used to decide weight values to obtain tradeoff optimal control inputs.

3.2. Nonparametric Estimation of Objective Function. In this section, two nonparametric estimation approaches are proposed to estimate the objective function (7).

(1) Kernel Density Estimation Method. The PDF of the tracking errors can be estimated from sample sequences $\left\{e_{i k}^{(1)}, e_{i k}^{(2)}, \ldots, e_{i k}^{(N)}\right\}(i=1,2)$ using Kernel density estimation (KDE) method:

$$
\begin{aligned}
\gamma_{e_{i k}}\left(\tau_{i}\right) \cong \widehat{\gamma}_{e_{i k}}\left(\tau_{i}\right)=\frac{1}{N} \sum_{j=1}^{N} \kappa\left(\tau_{i}-e_{i k}^{(j)}, \sigma_{i}^{2}\right) \quad(i=1,2), \\
\gamma_{e_{1 k} e_{2 k}}\left(\tau_{1}, \tau_{2}\right) \cong \widehat{\gamma}_{e_{1 k} e_{2 k}}\left(\tau_{1}, \tau_{2}\right) \\
=\frac{1}{N} \sum_{j=1}^{N} \kappa\left(\tau_{1}-e_{1 k}^{(j)}, \sigma_{1}^{2}\right) \kappa\left(\tau_{2}-e_{2 k}^{(j)}, \sigma_{2}^{2}\right),
\end{aligned}
$$

where $\kappa\left(x, \sigma^{2}\right)=(1 / \sqrt{2 \pi} \sigma) \exp \left(-x^{2} / 2 \sigma^{2}\right)$ is the Gaussian Kernel function with standard deviation $\sigma$.

According to definitions, the estimation of quadratic Renyi's entropy and mean value can be formulated as

$$
H\left(e_{i k}\right) \cong-\log \frac{1}{N^{2}} \sum_{j=1}^{N} \sum_{l=1}^{N} \kappa\left(e_{i k}^{(j)}-e_{i k}^{(l)}, 2 \sigma_{i}^{2}\right) \quad(i=1,2),
$$




$$
\begin{aligned}
& H\left(e_{1 k}, e_{2 k}\right) \\
& \cong-\log \frac{1}{N^{2}} \sum_{j=1}^{N} \sum_{l=1}^{N} \kappa\left(e_{1 k}^{(j)}-e_{1 k}^{(l)}, 2 \sigma_{1}^{2}\right) \kappa\left(e_{2 k}^{(j)}-e_{2 k}^{(l)}, 2 \sigma_{2}^{2}\right) \\
& E\left(e_{i k}^{2}\right) \cong \frac{1}{N} \sum_{j=1}^{N}\left(e_{i k}^{(j)}\right)^{2} \quad(i=1,2)
\end{aligned}
$$

Substituting (9a)-(9c) into (7), the single objective function then can be obtained.

Remark 2. Kernel density estimation method is verified to be an effective PDF estimation approach. However, $N$ samples are generated at each instant $k$, which requires mass memory and results in heavy computing burden.

(2) Sliding Window Technique. To enhance the computing efficiency, an alternative method called sliding window technique is proposed here.

At instant $k$, quadratic Renyi's entropy can be rewritten as

$$
\begin{array}{r}
H\left(e_{i k}\right)=-\log \int_{a_{e_{i}}}^{b_{e_{i}}} \gamma_{e_{i k}}^{2}\left(\tau_{i}\right) d \tau_{i}=-\log \left(E\left[\gamma_{e_{i k}}\left(\tau_{i}\right)\right]\right) \\
(i=1,2),
\end{array}
$$

$$
\begin{aligned}
H\left(e_{1 k}, e_{2 k}\right) & =-\log \int_{a_{e_{1}}}^{b_{e_{1}}} \int_{a_{e_{2}}}^{b_{e_{2}}} \gamma_{e_{1 k} e_{2 k}}^{2}\left(\tau_{1}, \tau_{2}\right) d \tau_{2} d \tau_{1}, \\
& =-\log \left(E\left[\gamma_{e_{1 k} e_{2 k}}\left(\tau_{1}, \tau_{2}\right)\right]\right) .
\end{aligned}
$$

Drop the expectation and use the most current sample of tracking error in the PDF to obtain the following stochastic estimate for entropies:

$$
\begin{gathered}
H\left(e_{i k}\right)=-\log \left(E\left[\gamma_{e_{i k}}\left(\tau_{i}\right)\right]\right) \approx-\log \left(\gamma_{e_{i k}}\left(\tau_{i}\right)\right), \\
H\left(e_{1 k}, e_{2 k}\right) \approx-\log \left(E\left[\gamma_{e_{1 k} e_{2 k}}\left(\tau_{1}, \tau_{2}\right)\right]\right) \\
=-\log \left(\gamma_{e_{1 k} e_{2 k}}\left(\tau_{1}, \tau_{2}\right)\right),
\end{gathered}
$$

where $e_{i k}(i=1,2)$ denote the most recent samples of tracking error at instant $k$. Next, the "sliding window" technology is employed to estimate the (joint) PDF of tracking error over the most recent $L$ samples $\left\{e_{i, k-L}, e_{i, k-L+1}, \ldots, e_{i, k-1}\right\}$. When $k<L$, the needed data can be complemented according to the history data of the system. Then, the estimation of (joint) $\mathrm{PDF}$ is given by

$$
\begin{gathered}
\gamma_{e_{i k}}\left(e_{i k}\right)=\frac{1}{L} \sum_{j=k-L}^{k-1} \kappa\left(e_{i k}-e_{i j}, \sigma_{i}\right), \quad(i=1,2), \\
\gamma_{e_{1 k} e_{2 k}}\left(e_{1 k}, e_{2 k}\right)=\frac{1}{L} \sum_{j=k-L}^{k-1} \kappa\left(e_{1 k}-e_{1 j}, \sigma_{1}\right) \kappa\left(e_{2 k}-e_{2 j}, \sigma_{2}\right) .
\end{gathered}
$$

Thus the stochastic estimate of the (joint) entropy of tracking error at instant $k$ becomes

$$
\begin{gathered}
H\left(e_{i k}\right)=-\log \left(\frac{1}{L} \sum_{j=k-L}^{k-1} \kappa\left(e_{i k}-e_{i j}, \sigma_{i}\right)\right), \quad(i=1,2), \\
H\left(e_{1 k}, e_{2 k}\right)=-\log \frac{1}{L} \sum_{j=k-L}^{k-1} \kappa\left(e_{1 k}-e_{1 j}, \sigma_{1}\right) \kappa\left(e_{2 k}-e_{2 j}, \sigma_{2}\right) .
\end{gathered}
$$

3.3. Neural Network Controller. Based on the known information $I_{k}=\left\{y_{k}, y_{k-1}, \ldots, y_{k-M} ; u_{k-1}, u_{k-2}, \ldots, u_{k-N} ; r_{k}\right\}(M$ and $N$ are numbers of previous measurements and controls), a neural network with fixed number of layers and nodes is used in this paper to directly model the resulting unknown control law without the need to construct a separate model for the unknown WECS dynamics. Here, the output of the $\mathrm{NN}$ will correspond to the value of the control $u_{k}$. Associated with the NN producing $u_{k}$ will be a vector of connection weights $\theta_{k} \in R^{p}$ that should be trained. Therefore, the control problem in this paper is equivalent to finding $\theta_{k}$ to minimize the performance index (7). And after the optimal $\theta_{k}$ has been found, the optimal control $u_{k}$ would be the output of neural network.

As a result, in theory, the training of $\theta_{k}$ can be obtained by minimizing $J_{k}$ to give

$$
\theta_{k}=\theta_{k-1}-\left.\alpha_{k} \frac{\partial J_{k}}{\partial \theta_{k}}\right|_{\theta_{k}=\theta_{k-1}} .
$$

However, the gradient-descent-type algorithms are not generally feasible in the model-free setting here. As such, the above algorithm can only be regarded as a guideline for the theoretical inside into the training scheme. Motivated by the method in [21], the following steps should be employed.

(1) Set the current sample time as $k-1$, and fix $\theta_{k-1}^{0}=$ $\left[\begin{array}{llll}\theta_{1, k-1}^{0} & \theta_{2, k-1}^{0} & \cdots & \theta_{p, k-1}^{0}\end{array}\right]^{T}$.

(2) According to the fixed neural network used in this paper, the control input $u_{k-1}$ can be calculated.

(3) Calculate the performance function as $J\left(\theta_{k-1}^{0}\right)$ according to (7).

(4) Disturb $\theta_{k-1}^{0}$ by $\Delta \theta_{k-1}$ and use the new $\theta$-value $\theta_{k-1}^{\text {new }}=$ $\theta_{k-1}^{0}+\Delta \theta_{k-1}$ to generate another control input $u_{k-1}^{\text {new }}$.

(5) Calculate the related performance function as $J\left(\theta_{k-1}^{0}+\right.$ $\left.\Delta \theta_{k-1}\right)$ using (7).

(6) Formulate the required gradient vector in (14):

$$
\left.\frac{\partial J_{k}}{\partial \theta_{k}}\right|_{\theta_{k}=\theta_{k-1}^{0}} \approx \frac{J\left(\theta_{k-1}^{0}+\Delta \theta_{k}\right)-J\left(\theta_{k-1}^{0}\right)}{\Delta \theta_{k}} .
$$

(7) Calculate $\theta_{k}$ based on (14). 
TABLE 1

\begin{tabular}{lcc}
\hline Parameters & Description & Numerical value \\
\hline$P_{g, \text { rat }}$ & Rated generator power & $2.0 \mathrm{MW}$ \\
$\tau$ & Time constant of the pitch system & $0.1 \mathrm{~s}$ \\
$\tau_{g}$ & Time constant of the generator & $20 \mathrm{~ms}$ \\
$J_{g}$ & system & \\
$J_{t}$ & Inertia of the generator & $56.29 \mathrm{~kg} \cdot \mathrm{m}^{2}$ \\
$B_{s}$ & Inertia of the turbine & $1.86 \times 10^{6}$ \\
$\omega_{g, \text { rat }}$ & Shaft damping coefficients & 212.2 \\
$i$ & Rated generator speed & $220 \mathrm{rad} / \mathrm{s}$ \\
$R$ & Gear ratio & 74.38 \\
$\rho$ & Blade length of the wind turbine & $33 \mathrm{~m}$ \\
\hline
\end{tabular}

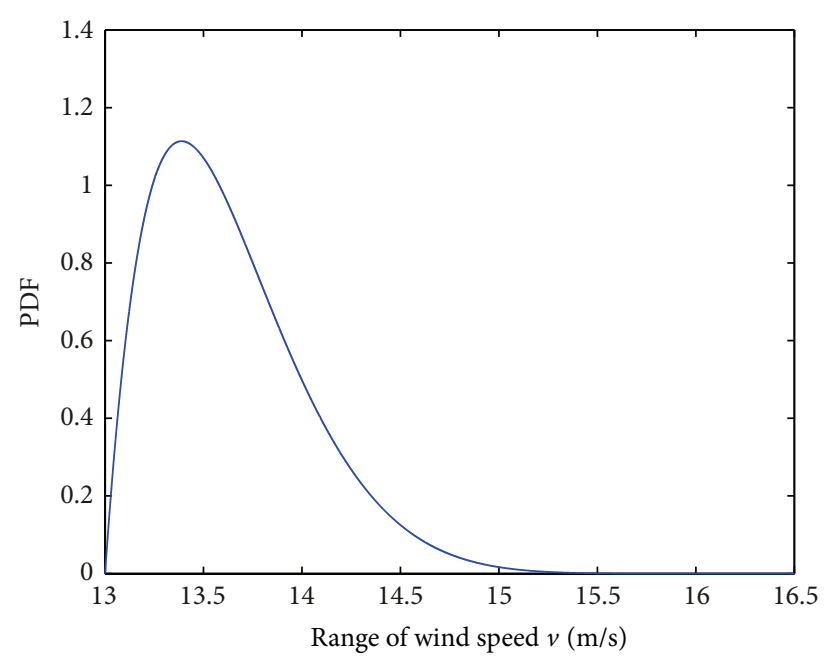

Figure 3: PDF of wind speed.

Note that every time when the dataset is generated, the vectors $\left\{I_{k}\right\}$ need to be updated as well.

Based on the obtained $\theta_{k}$, the optimal control input can be calculated easily.

Remark 3. The estimation of gradient vector $\left.\left(\partial J_{k} / \partial \theta_{k}\right)\right|_{\theta_{k}=\theta_{k-1}}$ in (14) can be obtained by using the simultaneous perturbation stochastic approximation approach in [22]. And the convergence of this method also can be found in [23].

\section{Simulation Results}

In this section, the proposed control method is applied to a certain variable-speed variable-pitch WECS. The model in (1)-(4) is used for producing the measurements. Model parameters are shown in Table 1.

The simulation is carried out on the basis of the working condition with $\omega_{g}=150 \mathrm{rad} / \mathrm{s}$ and $P_{g}=1.5 \mathrm{MW}$. The PDF of wind speed is given in Figure 3. The sampling period is $T_{s}=1 \mathrm{~s}$, sliding window width is $L=100$, and forgetting factor is $\lambda=0.0095$. The weights in (7) are $R_{1}=0.1, R_{2}=0.1$,

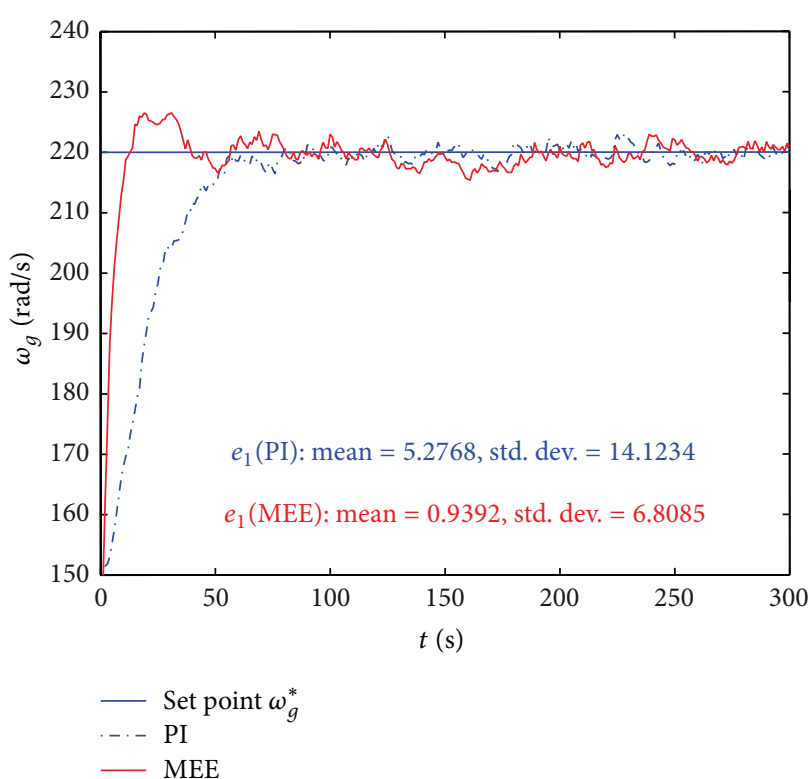

FIGURE 4: Responses of generator speed.

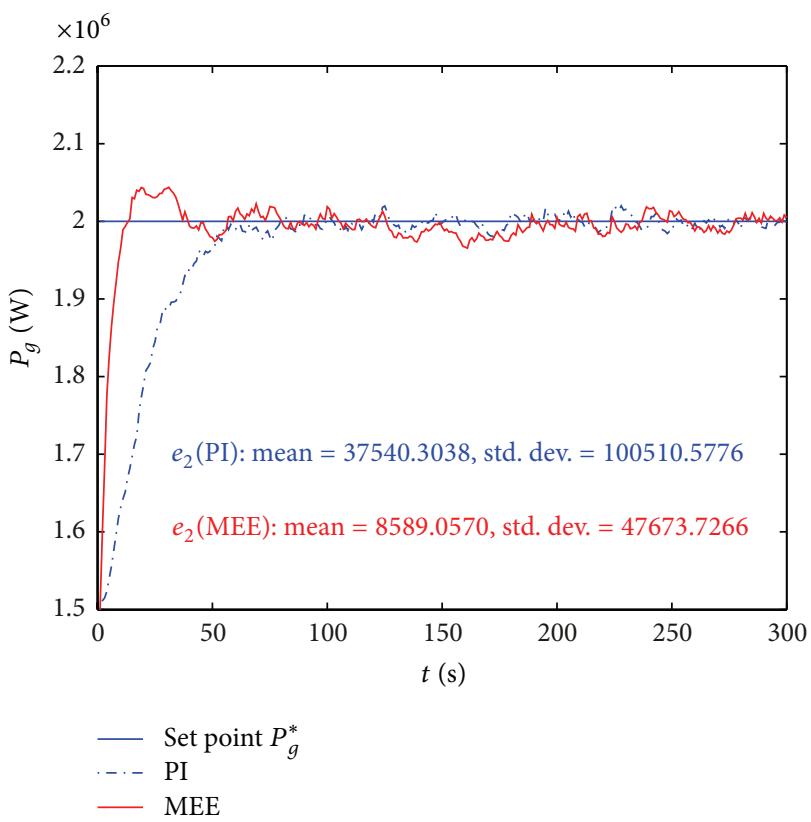

FIGURE 5: Responses of generator power.

$R_{3}=0.001, R_{4}=0.001, R_{5}=10$, and $R_{6}=\left[\begin{array}{lll}10^{-4} & 10^{-5} \\ 10^{-5} & 10^{-3}\end{array}\right]$. The controller is modeled by using a NN with two layers, one of 20 nodes and one of 10 nodes [23]. The inputs to the controller include the current and most recent output, the most recent control, and the current set point, yielding a total of eight input nodes. Therefore, the total number of weights to be trained is 412 .

The advantage of the proposed method is shown by comparing with PI controllers whose optimal PI parameters are tuned using the MATLAB NCD toolbox. Transfer functions of rotary speed controller and power controller are $G_{C 1}(s)=$ 

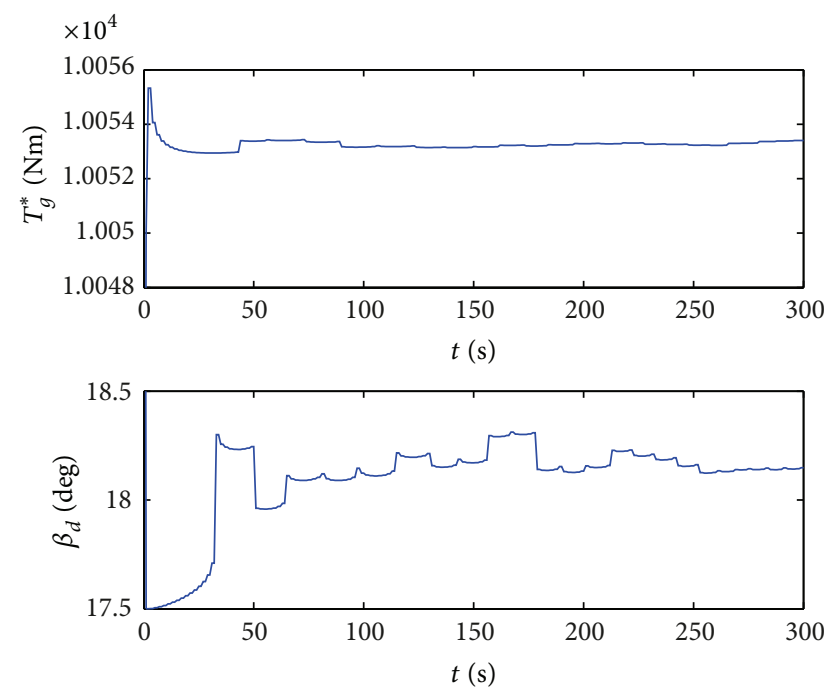

FIgURE 6: Control inputs.

$0.0015+0.045 / s$ and $G_{C 2}(s)=0.095+0.058 / s$, respectively. The comparative results are shown in Figures 4, 5, 6, 7, 8, and 9.

From Figures 4 and 5, it can be seen that, compared with the conventional PI control strategy, the proposed control method can make generator speed and power have smaller fluctuations. Small oscillation of the generator speed can reduce the mechanical load of wind turbines, corresponding to a reliable operation of the wind power system. On the other hand, small fluctuation of the generator power can guarantee more stable supply power and consumers' required power quality. The variation of control inputs is presented in Figure 6. It is clear that the changes of electromagnetic torque and pitch angle are smooth. In Figure 7, the objective function (7) is decreasing with time and finally approaching a small value, and this means that the WECS can achieve a satisfying performance under the proposed control.

The shapes of PDFs of generator speed and power in (8a) and $(8 \mathrm{~b})$ become narrower and sharper along with sampling time using the proposed control method; this indicates that the WECS has a small uncertainty in its closed-loop operation. It also can be verified from Figure 9, in which the PDFs of generator speed and power at several typical instants are shown.

The above simulation results illustrate that the proposed control approach can obtain better performance over the PI controller.

\section{Conclusions}

In this paper, a neural network controller design approach in the data-driven framework is proposed for the wind energy conversion system (WECS). The proposed method differs from previous results in minimum entropy control: this method avoids the construction of a system model and focuses directly on regulating the WECS via the construction of a closed-loop control algorithm based on a neural network

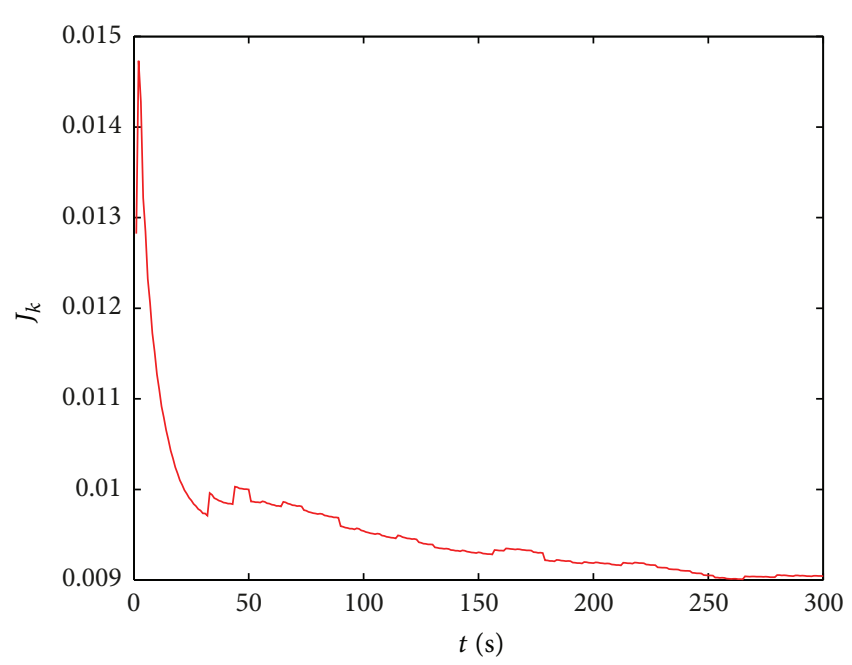

Figure 7: Performance index.

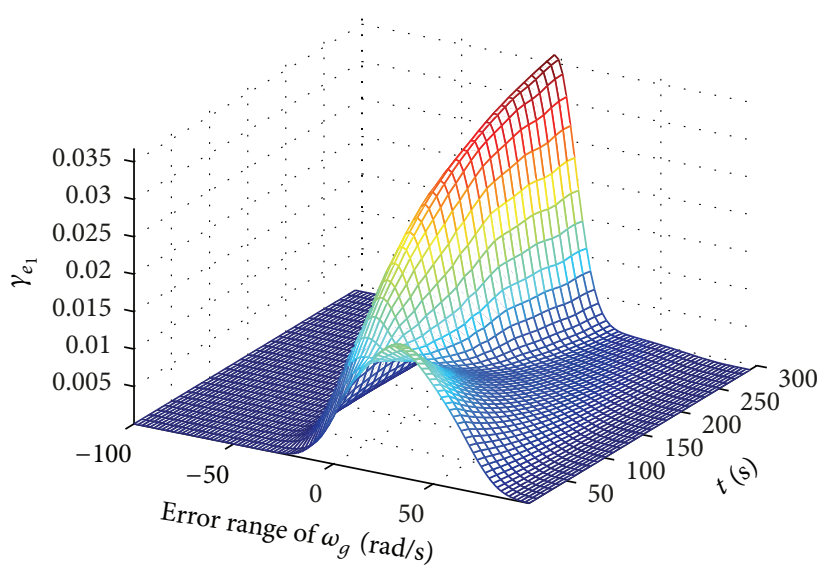

(a)

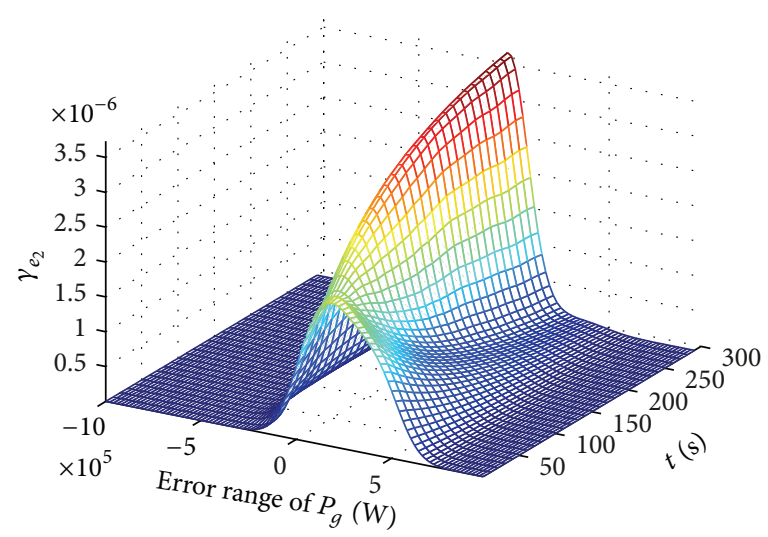

(b)

FIGURE 8: 3D PDFs of tracking error: (a) generator speed; (b) generator power.

with fixed structure. Since there are no assumption equations describing the WECS, it is not possible to calculate the gradient of the objective function for use in standard gradientdescent-type search algorithms. Therefore, an incremental perturbation approximation method is proposed to estimate the gradient. The proposed approach is applied to a certain 


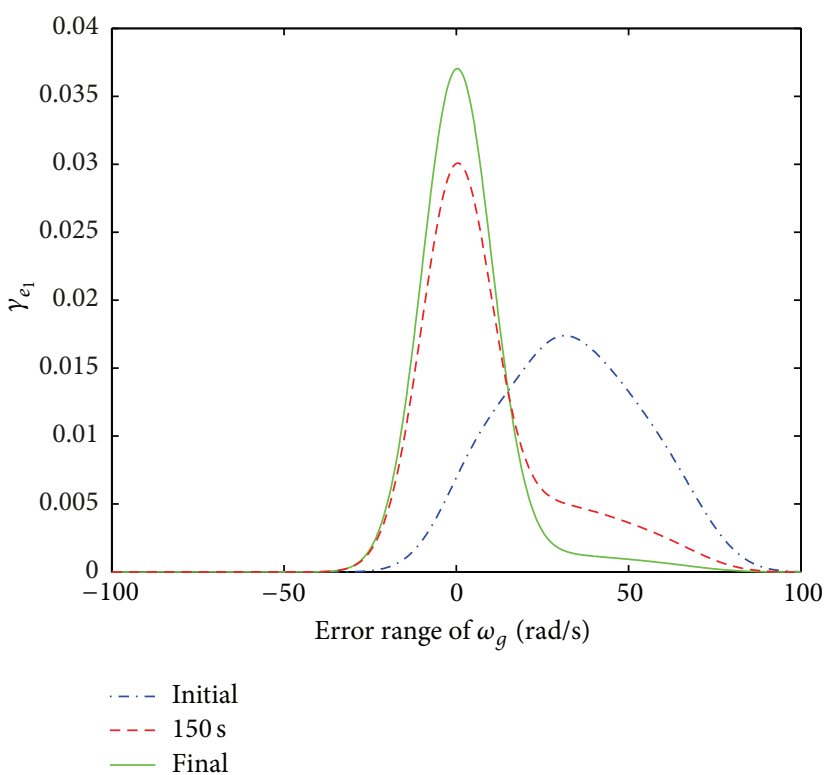

(a)

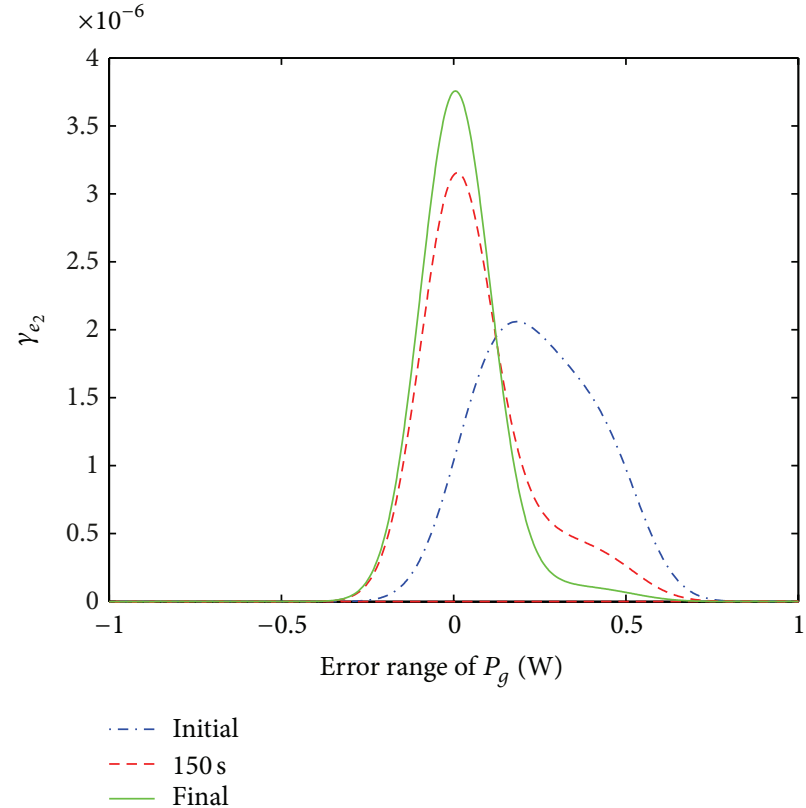

(b)

FIGURE 9: PDFs of tracking error at typical instants: (a) generator speed; (b) generator power.

WECS where the control objective is to track the target values for both generator speed and output power as closely as possible. Simulation results show that the proposed control method can achieve good performance.

\section{Nomenclature}

$\begin{array}{ll}\omega_{t}: & \text { Speed of the low-speed shaft } \\ \omega_{g}: & \text { Generator speed } \\ T_{t w}: & \text { Flexible shaft } \\ T_{g}: & \text { Generator torque } \\ \beta_{:} & \text {Pitch angle } \\ T_{g}^{*}: & \text { Generator torque set point } \\ \beta_{d}: & \text { Desired pitch angle } \\ P_{g}: & \text { Generator power } \\ J_{t}: & \text { Inertia of the turbine } \\ J_{g}: & \text { Inertia of the generator } \\ v: & \text { Wind speed } \\ i: & \text { Gear ratio } \\ \lambda: & \text { Tip speed ratio } \\ C_{p}(\lambda, \beta): \text { Power coefficient } \\ \rho: & \text { Air density } \\ k_{s}: & \text { Shaft stiffness coefficients } \\ B_{s}: & \text { Shaft damping coefficients } \\ R: & \text { Blade length of the wind turbine } \\ \tau_{g}: & \text { Time constant of the generator system } \\ P_{t}: & \text { Harvested mechanical power of the wind turbine } \\ \tau: & \text { Time constant of the pitch system. }\end{array}$

\section{Conflict of Interests}

The authors declare no conflict of interests.

\section{Acknowledgments}

This work was supported by the National Basic Research Program of China under Grant 973 Program 2011 CB710706 and China National Science Foundation under Grant 61374052. These are gratefully acknowledged.

\section{References}

[1] A. Kusiak, W. Li, and Z. Song, "Dynamic control of wind turbines," Renewable Energy, vol. 35, no. 2, pp. 456-463, 2010.

[2] J. K. Kaldellis, "The wind potential impact on the maximum wind energy penetration in autonomous electrical grids," Renewable Energy, vol. 33, no. 7, pp. 1665-1677, 2008.

[3] M. M. Hand and M. J. Balas, Non-Linear and Linear Model Based Controller Design for Variable-Speed Wind Turbines, National Renewable Energy Laboratory, 1999.

[4] T. Knudsen, P. Andersen, and S. Toffner-Clausen, "Comparing PI and robust pitch controllers on a $400 \mathrm{~kW}$ wind turbine by full-scale tests," in Proceeding of the European Wind Energy Conference, pp. 546-550, Dublin, Ireland, 1997.

[5] T. Ekelund, Modeling and linear quadratic optimal control of wind turbines [Ph.D. thesis], Chalmers University of Technology, Gothenburg, Sweden, 1997.

[6] I. Munteanu, N. A. Cutululis, A. I. Bratcu, and E. Ceangă, "Optimization of variable speed wind power systems based on a LQG approach," Control Engineering Practice, vol. 13, no. 7, pp. 903-912, 2005.

[7] B. Boukhezzar and H. Siguerdidjane, "Nonlinear control of variable speed wind turbines for power regulation," in Proceedings of the IEEE Conference on Control Applications, pp. 114-119, Toronto, Canada, 2005.

[8] E. B. Muhando, T. Senjyu, A. Yona, H. Kinjo, and T. Funabashi, "Disturbance rejection by dual pitch control and self-tuning 
regulator for wind turbine generator parametric uncertainty compensation," IET Control Theory and Applications, vol. 1, no. 5, pp. 1431-1440, 2007.

[9] B. Beltran, M. E. H. Benbouzid, and T. Ahmed-Ali, "Secondorder sliding mode control of a doubly fed induction generator driven wind turbine," IEEE Transactions on Energy Conversion, vol. 27, no. 2, pp. 261-269, 2012.

[10] S. Bououden, O. Benelmir, S. Ziani, and S. Filali, "A new adaptive fuzzy model and output terminal constraints in predictive control," International Journal of Information Systems Sciences, vol. 3, no. 1, pp. 25-35, 2007.

[11] M. Soliman, O. P. Malik, and D. T. Westwick, "Multiple model predictive control for wind turbines with doubly fed induction generators," IEEE Transactions on Sustainable Energy, vol. 2, no. 3, pp. 215-225, 2011.

[12] F. D. Bianchi, R. J. Mantz, and C. F. Christiansen, "Power regulation in pitch-controlled variable-speed WECS above rated wind speed," Renewable Energy, vol. 29, no. 11, pp. 19111922, 2004.

[13] W. Lin, C. Hong, and F. Cheng, "Fuzzy neural network output maximization control for sensorless wind energy conversion system," Energy, vol. 35, no. 2, pp. 592-601, 2010.

[14] M. Soliman, O. P. Malik, and D. T. Westwick, "Fault tolerant control of variable-speed variable-pitch wind turbines: a subspace predictive control approach," in Proceedings of the 16th IFAC System Identification Symposium, pp. 1683-1688, July 2012.

[15] G. van der Veen, Identification of wind energy systems [Ph.D. thesis], Technische Universiteit Delft, 2013.

[16] F. Lescher, J. Y. Zhao, and A. Martinez, "LQG multiple model control of a variable speed pitch regulated wind turbine," in Proceedings of the 17th IMACS World Congress, Paris, France, 2005.

[17] A. Pintea, N. Christov, D. Popescu, and P. Borne, "LQG control of horizontal wind turbines for blades and tower loads alleviation," in Proceedings of the 18th of the World Congress International Federation of Automatic Control (IFAC '11), pp. 1721-1726, September 2011.

[18] S. Nourdine, H. Camblong, I. Vechiu, and G. Tapia, "Comparison of wind turbine LQG controllers using individual pitch control to alleviate fatigue loads," in Proceedings of the 18th Mediterranean Conference on Control and Automation (MED '10), pp. 1591-1596, Marrakech, Morocco, June 2010.

[19] P. Afshar, H. Wang, and T. Chai, "An ILC-based adaptive control for general stochastic systems with strictly decreasing entropy," IEEE Transactions on Neural Networks, vol. 20, no. 3, pp. 471482, 2009.

[20] J. H. Zhang, C. C. Chu, J. Munoz, and J. H. Chen, "Minimum entropy based run-to-run control for semiconductor processes with uncertain metrology delay," Journal of Process Control, vol. 19, no. 10, pp. 1688-1697, 2009.

[21] H. Wang and X. Sun, "Neural network based probability density function shape control for unknown stochastic systems," in Proceedings of the IEEE International Symposium on Intelligent Control (ISIC '04), pp. 120-125, Taipei, Taiwan, September 2004.

[22] J. C. Spall and J. A. Cristion, "A neural network controller for systems with unmodeled dynamics with applications to wastewater treatment," IEEE Transactions on Systems, Man, and Cybernetics B, vol. 27, no. 3, pp. 369-375, 1997.

[23] J. C. Spall and J. A. Cristion, "Model-free control of nonlinear stochastic systems with discrete-time measurements," IEEE Transactions on Automatic Control, vol. 43, no. 9, pp. 1198-1210, 1998.
[24] M. Soliman, O. P. Malik, and D. T. Westwick, "Multiple model multiple-input multiple-output predictive control for variable speed variable pitch wind energy conversion systems," IET Renewable Power Generation, vol. 5, no. 2, pp. 124-136, 2011.

[25] A. D. Hansen, P. Sørensen, F. Lov, and F. Blaabjerg, "Control of variable speed wind turbines with doubly-fed induction generators," Wind Engineering, vol. 28, no. 4, pp. 411-432, 2004.

[26] F. Wu, X. P. Zhang, K. Godfrey, and P. Ju, "Small signal stability analysis and optimal control of a wind turbine with doubly fed induction generator," IET Generation, Transmission and Distribution, vol. 1, no. 5, pp. 751-760, 2007.

[27] J. C. Principe, Information Theoretic Learning: Renyi's Entropy and Kernel Perspectives, Information Science and Statistics, Springer, New York, NY, USA, 2010. 


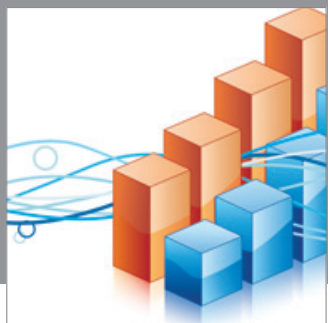

Advances in

Operations Research

mansans

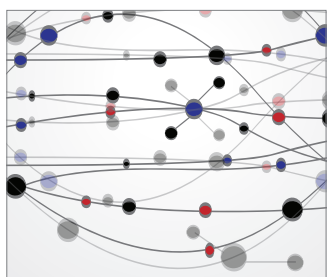

The Scientific World Journal
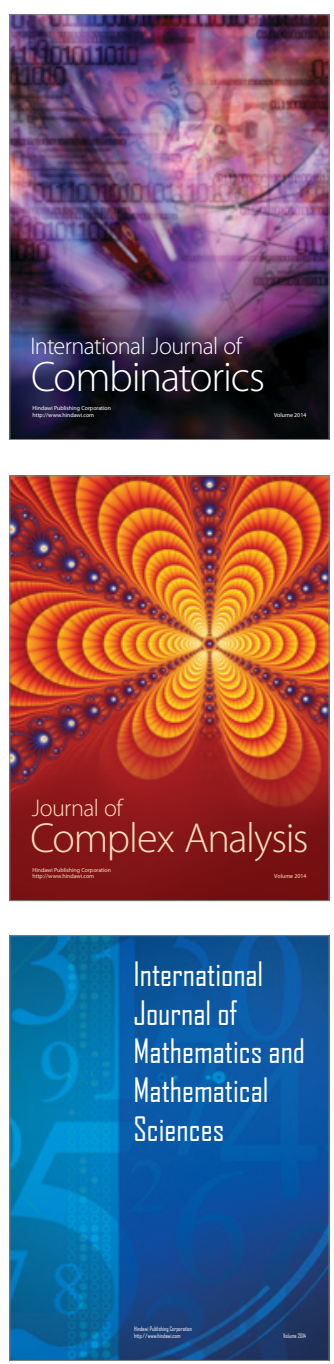
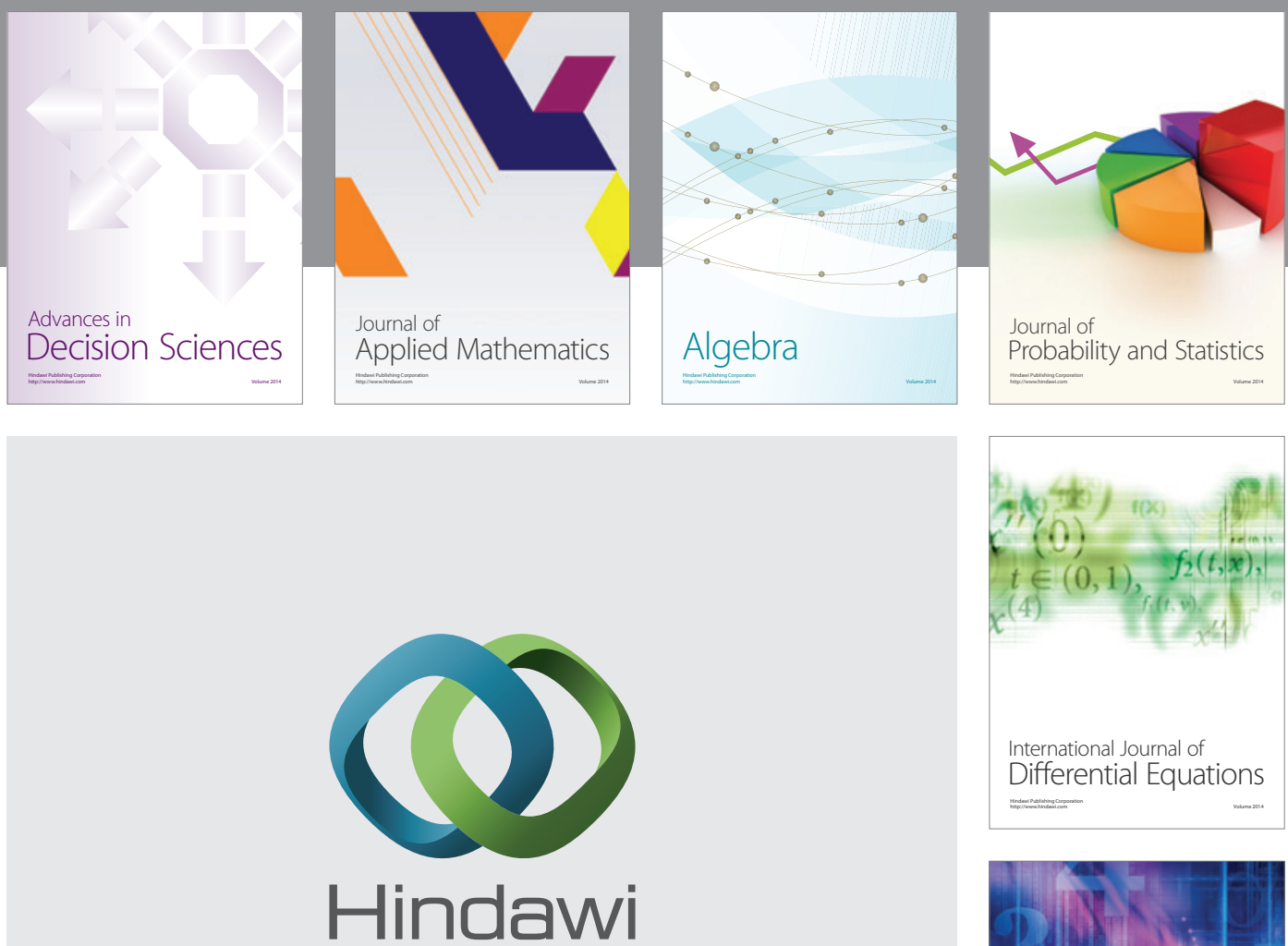

Submit your manuscripts at http://www.hindawi.com
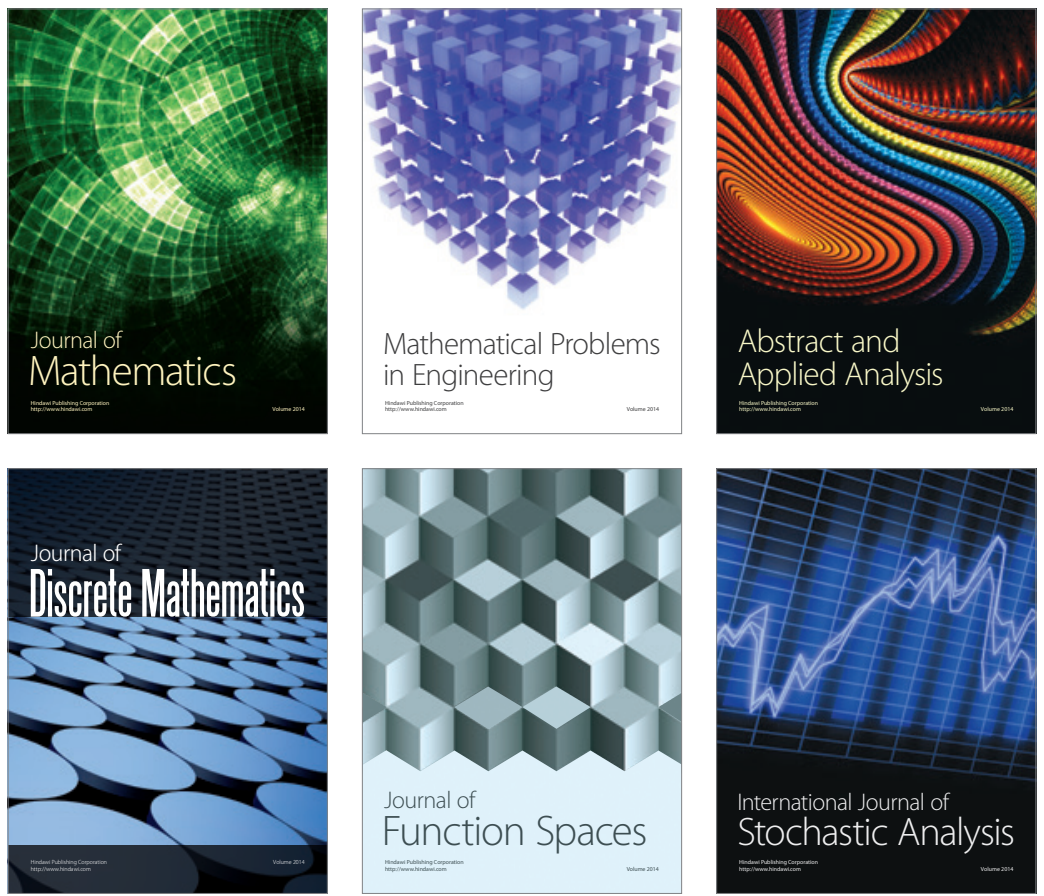

Journal of

Function Spaces

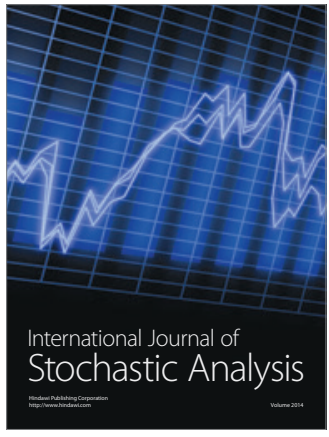

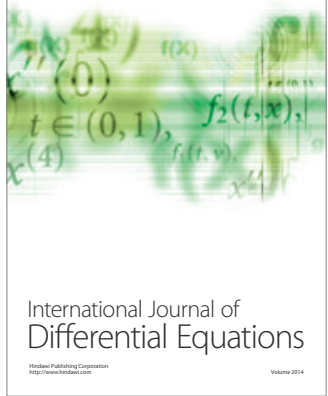
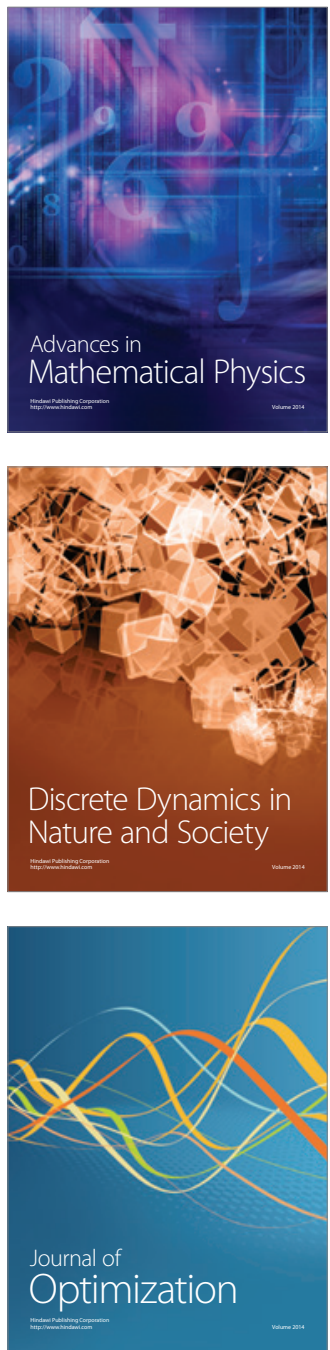